\title{
Network Structure and Knowledge Transfer
}

\author{
Fangcheng Tang ${ }^{1,2}$ \\ ${ }^{1}$ School of Economics and Management, Tsinghua University \\ Beijing 100084, P.R. China \\ ${ }^{2}$ School of Economics and Management, Xi'an Technological University \\ Xi'an 710032, P.R. China \\ fctang@163.com
}

\begin{abstract}
This study employs single layer perceptron model (SLPM) to explore how the topological structure of intra-organization networks affects knowledge transfer. The results demonstrate that in the process of knowledge transfer, both the disseminative capacity of knowledge senders and the absorptive capacity of knowledge receivers should be taken into consideration. While hierarchical networks can enable greater numbers of organizational units to acquire knowledge, they reduce the speed and efficiency of knowledge transfer, whereas scale-free networks can accelerate transfer of knowledge among units.
\end{abstract}

Keywords: Knowledge transfer, Hierarchical network, Scale-free network.

\section{Introduction}

This paper utilizes a single-layer perceptron neural network model (SLPM) [1][2] to simulate the knowledge transfer process in two network topologies of an organization. Specifically, our study focuses on the potentially different performance of knowledge transfer between two organizational network topologies: hierarchical and scale-free network which represent informal and formal structures in an organization. We argue that the nature of knowledge transfer within intra-organizational networks can be modeled with the tools of computational learning theory (CLT) as developed by computer scientists to study learning and problem-solving by machines. As the knowledge is transferred within an intra-organizational network, network nodes learn from their senders and become proficient at recognizing new and related knowledge.

\section{Virtual Experiment Design}

\subsection{The Structure of Intra-organization Networks}

An intra-organization network can be described by a graph theoretic approach in this form: $G=(V, E)$, where $V$ denotes the set of units and $E$ is sets of the relationships in the networks.Let $V=1, \ldots, N$ denote a finite set of units.for $\forall i, j \in V$, 
define the binary varible $\chi(i, j)$ taking value $\chi(i, j)=1$ if there is a connection between $i$ and $j$, otherwise $\chi(i, j)=0$.

Thus, a symmetrical hierarchical structure for vertical organizations where the number of layers, the number of nodes in the first layer and the number of connections from each node to the next layer are set. A scale-free model which is defined by the Watts and Strogatz [3]. The network evolves into a scale-invariant state, the probability that a vertex has $\mathrm{k}$ edges following a power law with an exponent $\gamma_{\text {model }}=2.9 \pm 0.1[4]$.

\subsection{Node Status Variables}

We assuming that individual in the organization can be characterized by three variables $\alpha, \beta, \rho$. The first is a signal variable $\alpha$, which demonstrates whether individual acquired specific knowledge, and value 1 means the individual successfully gets access to the knowledge and 0 means the opposite. The second variable $\beta$, which is independent randomized uniformly distributed on $[0,1]$, represents the capacity of the individuals to learn and absorb specific knowledge. Larger values mean substantial capacity, which can be determined by the genetics, background, or experience of these members. The last variable $\rho$ shows the disseminative capacity of one member to transfer knowledge to others. Variable $\rho$ is also random uniformly distributed on $[0,1]$ and members who have strong capacity to spread knowledge get high numerical values.

\subsection{Interaction Mechanism and NN Model}

Here we use the SLPM of neural network to simulate the knowledge transfer. Every node in the network is regarded as a threshold logical cell, input $X=$ $\left(X_{1}, X_{2}, \ldots, X_{N}\right)$, each $X_{i}$ equals to the output $\Omega_{i}$ of vertex $i$, shows the variable $S$ of node $i, W=\left\{\omega_{1 j}, \omega_{2 j}, \ldots, \omega_{v j}\right\}$ and $\omega_{i j}$ comes from the variable $F_{i}, \theta$ is the threshold value which is derived from the ingredients variable $D$ of the node. This model can be interpreted as that a node which has difficulty $D$ in comprehending the knowledge can only learn the knowledge under the condition that the sum of the $F$ of the input node which already has the knowledge is bigger than $D$ of this node, so output $\Omega_{i}=\{0,1\}$ is calculated as:

$$
\Omega_{j}=f\left(\sum_{i=1}^{n} \omega_{i j} X_{i}-\theta_{i}\right), f\left(u_{i}\right)= \begin{cases}0 & \text { if } \sigma_{i} \leq 0 \\ 1 & \text { if } \sigma_{i}>0\end{cases}
$$

\section{Results}

A comparison of results of the computational experiments is summarized in Fig. 1 and Fig.2. In Fig1 (a) and (b), as the disseminative capacity of the dominant individual is set at 1 , the average number of knowledge-acquired individuals is increased, and the number of knowledge-acquired nodes is more with higher 
absorptive capacity (dashed line) than that with lower absorptive capacity (solid line) in hierarchical and scale-free network. Regardless of high or low disseminative capacity, the frequency of KSC tends to increase at first and then decline as the number of knowledge-acquired individuals increases. But the extent of frequency of KSC increases with low absorptive capacity is less than that with high absorptive. High absorptive capacity enables the number of knowledge-acquired individuals to increase more, and it seems that the frequency of scale-free network follows the assumption of normal distribution.

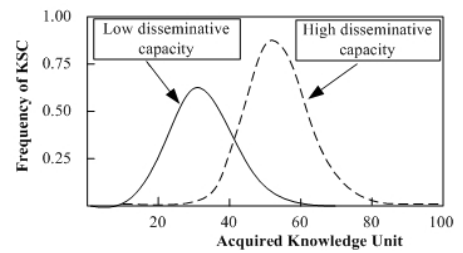

(a) hierarchical network

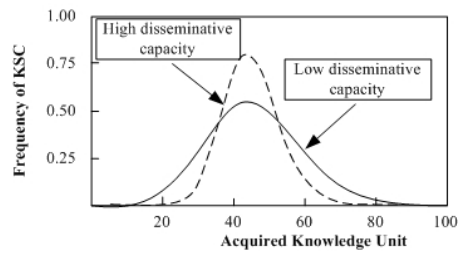

(b) scale-free network

Fig. 1. The relationship between the frequency of KSC and acquired knowledge member with different disseminative capabilities of knowledge holders

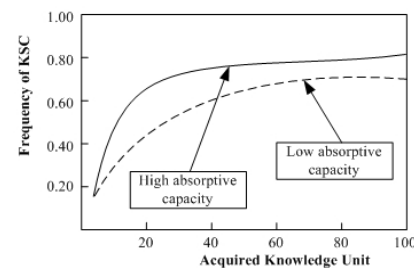

(a) Hierarchical Network

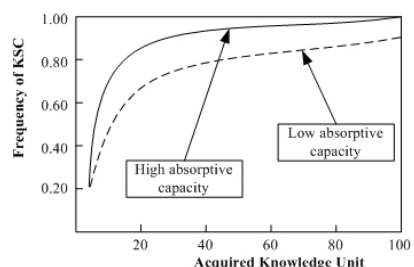

(b) Scale-free Network

Fig. 2. Relationship between the frequency of KSC and acquired knowledge member with absorptive capacity

Fig. 2 shows that, regardless of the higher or lower absorptive capacity, the frequency of KSC of knowledge-acquired units in hierarchical and scale-free network increases. Simultaneously, the extent of frequency of KSC with high absorptive capacity increases is more than that with low absorptive capacity. In hierarchical networks, average knowledge state change with high absorptive capacity tends to be stable at interval $0.60 \sim 0.80$. In scale-free networks, however, the tendency to increase will reach the maximum value 1.00. In addition, the frequency of KSC with low absorptive capacity in scale-free networks tends to be more than that of hierarchical networks. Therefore, as an important channel to knowledge transfer, scale-free networks enable knowledge transfer to be more efficient than does hierarchical networks. 


\section{Conclusions}

This study demonstrated that efficient and effective knowledge transfer must consider two important aspects: knowledge senders' disseminative capacity and knowledge receivers' absorptive capacity. Cohen and Levinthal argue that organizations have to build specific absorptive capacity to identify, assimilate, and exploit external knowledge [5]. But an organizations also need to build strong disseminative capacity to communicate their knowledge in such a way that knowledge workers in the network can easily understand and thus assimilate the required knowledge, hence to maximize the value of knowledge. A corollary of this finding is to understand the role of the dominant knowledge holder in the knowledge transfer process. Also, an interesting effect of the model is that 100 per cent knowledge transfer in an intra-organizational network is never reached. We believe that this situation is the result of interaction of nodes with different disseminative capacity and absorptive capacity. The implication for multiunit organizations is that 100 per cent knowledge sharing in work-teams or groups is rarely achieved.

But current study has never considered the different types of knowledge, e.g., tacit and explict knowledge. In addition, the strength of ties between the players is not considered. These interesting problems will be remained as future research.

Acknowledgments. I would like to thank the anonymous reviewers for their insightful comments on previous versions of this manuscript.I was also grateful to the funding of National Science Fund of China under contract No. 70602004 and Postdoctoral Foundation of China under contract No. 2005038072 for their general support.

\section{References}

1. Hertz J., Krogh, A., Palmer, R. G. Introduction to the theory of neural computation. Redwood City: Addison-Wesley (1991).

2. Lippman, R. P. An introduction to computing with neural nets. IEEE ASSP Magazine 4 (1987) 4-22.

3. Watts, D.J., Strogatz, S. H. Collective dynamics of small world networks. Nature 393 (1998) 440-442.

4. Barabśsi, A-L., Albert, R., Jeong, H. Scale-free characteristics of random networks: the topology of the world-wide web. Physica A 281 (2000) 69-77.

5. Cohen, W., Levinthal, D. Absorptive capacity: A new perspective on learning and innovation. Administrative Science Quarterly 35 (1990) 128-152. 